\title{
Structural Revision of Garcinielliptin Oxide and Garcinielliptone E
}

Robert B. Grossman and Xing-Wei Yang

\section{Supporting Information}

Reported NMR assignments of garcinielliptin oxide (GO) and garcinielliptone E (GE) (Table S1), reported and redetermined NMR assignments of SE (Table S2), reacquired NMR spectra of SE (Figures S1-S9), MS and NMR spectra of GT (Figures S10-17), and NMR spectra of GN (Figures S18-19). 
Table S1. Reported ${ }^{13} \mathrm{C}$ and ${ }^{1} \mathrm{H}$ NMR Data of GO and GE (in $\mathrm{CDCl}_{3}$ ).

\begin{tabular}{|c|c|c|c|c|}
\hline \multirow{2}{*}{ no. } & \multicolumn{2}{|r|}{ GO } & \multicolumn{2}{|r|}{ GE } \\
\hline & $\delta \mathrm{C}$ & $\delta \mathrm{H}$, mult $(J$ in $\mathrm{Hz})$ & $\delta \mathrm{C}$ & $\delta \mathrm{H}$, mult $(J$ in $\mathrm{Hz})$ \\
\hline 1 & 209.0 & & 209.2 & \\
\hline 2 & 97.2 & & 97.1 & \\
\hline 3 & 206.9 & & 207.1 & \\
\hline 4 & 44.0 & & 44.0 & \\
\hline 5 & 107.9 & & 107.8 & \\
\hline 6 & 40.8 & $0.95, \mathrm{~m}$ & 40.8 & $0.87, \mathrm{~m}$ \\
\hline \multirow[t]{2}{*}{7} & 33.2 & $1.89, \mathrm{dd}(14.0,3.6)$ & 33.6 & $1.87, \mathrm{dd}(14.0,3.6)$ \\
\hline & & $1.25, \mathrm{dd}(14.0,10.0)$ & & $1.22, \mathrm{~m}$ \\
\hline 8 & 54.8 & & 54.9 & \\
\hline 9 & 70.6 & & 70.6 & \\
\hline 10 & 217.6 & & 217.3 & \\
\hline 11 & 38.7 & $3.20, \mathrm{~m}$ & 38.7 & $3.22, \mathrm{~m}$ \\
\hline 12 & 18.8 & $0.88, \mathrm{~d}(6.8)$ & 19.3 & $0.87, \mathrm{~d}(6.8)$ \\
\hline 13 & 17.0 & $1.09, \mathrm{~d}(6.8)$ & 16.9 & $1.09, \mathrm{~d}(6.8)$ \\
\hline 14 & 17.7 & $0.94, \mathrm{~s}$ & 17.7 & $0.93, \mathrm{~s}$ \\
\hline 15 & 23.0 & $1.23, \mathrm{~s}$ & 23.0 & $1.25, \mathrm{~s}$ \\
\hline \multirow[t]{2}{*}{16} & 23.7 & $2.74, \mathrm{dd}(15.6,6.8)$ & 19.8 & $2.32 \mathrm{dd}(13.4,4.4)$ \\
\hline & & $2.49, \mathrm{dd}(15.6,6.8)$ & & $1.71, \mathrm{dd}(13.4,4.4)$ \\
\hline \multirow[t]{2}{*}{17} & 116.0 & $5.01^{a}, \mathrm{t}(6.8)$ & 36.7 & $1.18, \mathrm{~m}$ \\
\hline & & & & $1.09, \mathrm{~m}$ \\
\hline 18 & 135.8 & & 70.2 & \\
\hline 19 & 25.8 & $1.64, \mathrm{~s}$ & 28.8 & $1.19, \mathrm{~s}$ \\
\hline 20 & 17.9 & $1.63, \mathrm{~s}$ & 29.4 & $1.22, \mathrm{~s}$ \\
\hline \multirow[t]{2}{*}{21} & 27.7 & 2.05, brd (12.0) & 27.7 & 2.07, brd (13.2) \\
\hline & & 1.55 , overlap & & $1.18, \mathrm{~m}$ \\
\hline 22 & 122.1 & $4.92, \mathrm{t}(7.2)$ & 122.0 & $4.91, \mathrm{t}(7.6)$ \\
\hline 23 & 133.3 & & 133.3 & \\
\hline 24 & 25.8 & $1.69, \mathrm{~s}$ & 25.8 & $1.67, \mathrm{~s}$ \\
\hline 25 & 17.9 & $1.55, \mathrm{~s}$ & 18.0 & $1.54, \mathrm{~s}$ \\
\hline 26 & 30.9 & $2.42^{a}, \mathrm{dd}(13.2,7.5)$ & 31.3 & $2.40, \mathrm{~d}(8.4)$ \\
\hline 27 & 118.9 & $5.27, \mathrm{t}(7.5)$ & 119.3 & $5.33, \mathrm{t}(7.2)$ \\
\hline 28 & 133.7 & & 133.3 & \\
\hline 29 & 26.0 & $1.69, \mathrm{~s}$ & 25.8 & $1.68, \mathrm{~s}$ \\
\hline 30 & 17.6 & $1.57, \mathrm{~s}$ & 17.7 & $1.55, \mathrm{~s}$ \\
\hline
\end{tabular}

${ }^{a}$ There were apparent typos in the original assignments (5.10 and 3.42), and the real chemical shifts of H17 and H26a of GO should be 5.01 and 2.42, respectively). 
Table S2. ${ }^{13} \mathrm{C}$ and ${ }^{1} \mathrm{H}$ NMR Data of Reported and Isolated SE in $\mathrm{C}_{6} \mathrm{D}_{6}$

\begin{tabular}{|c|c|c|c|c|}
\hline \multirow{2}{*}{ no. } & \multicolumn{2}{|c|}{ SE (reported) } & \multicolumn{2}{|r|}{ SE (isolated) } \\
\hline & $\delta \mathrm{C}$ & $\delta \mathrm{H}$, mult $(J$ in $\mathrm{Hz})$ & $\delta \mathrm{C}$ & $\delta \mathrm{H}$, mult $(J$ in $\mathrm{Hz})$ \\
\hline 1 & 209.0 & & 209.0 & \\
\hline 2 & 97.8 & & 97.8 & \\
\hline 3 & 207.4 & & 207.4 & \\
\hline 4 & 44.0 & & 44.0 & \\
\hline 5 & 108.4 & & 108.5 & \\
\hline 6 & 41.5 & $1.21, \mathrm{~m}$ & 41.5 & $1.21, \mathrm{~m}$ \\
\hline \multirow[t]{2}{*}{7} & 33.7 & $2.13, \mathrm{dd}(14.1,3.1)$ & 33.7 & $2.13, \mathrm{dd}(14.1,3.4)$ \\
\hline & & $1.31, \mathrm{t}(14.1)$ & & $1.31, \mathrm{t}(14.1)$ \\
\hline 8 & 55.2 & & 55.2 & \\
\hline 9 & 71.0 & & 71.0 & \\
\hline 10 & 218.1 & & 218.1 & \\
\hline 11 & 38.9 & $3.19, \mathrm{q}(6.6)$ & 38.9 & 3.18 , sept $(6.6)$ \\
\hline 12 & 18.8 & $0.78^{a}, \mathrm{~d}(6.6)$ & 18.8 & $0.78, d(6.6)$ \\
\hline 13 & 16.9 & $0.89, \mathrm{~d}(6.6)$ & 16.9 & $0.89, \mathrm{~d}(6.6)$ \\
\hline 14 & 17.6 & $0.85, \mathrm{~s}$ & 17.7 & $0.85, \mathrm{~s}$ \\
\hline 15 & 23.1 & $1.18, \mathrm{~s}$ & 23.1 & $1.18, \mathrm{~s}$ \\
\hline \multirow[t]{2}{*}{16} & 24.3 & $3.02, \mathrm{dd}(15.6,7.3)$ & 24.3 & $3.02, \mathrm{dd}(15.6,7.3)$ \\
\hline & & 2.75 , dd $(15.6,7.3)$ & & $2.74, \mathrm{dd}(15.6,7.3)$ \\
\hline 17 & 116.9 & $5.29, \mathrm{t}(7.3)$ & 116.9 & $5.29, \mathrm{t}(7.4)$ \\
\hline 18 & 135.7 & & 135.7 & \\
\hline 19 & 25.8 & $1.55, \mathrm{~s}$ & 25.8 & $1.54, \mathrm{~s}$ \\
\hline 20 & 17.8 & $1.53, \mathrm{~s}$ & 17.8 & $1.54, \mathrm{~s}$ \\
\hline \multirow[t]{2}{*}{21} & 28.0 & $1.94, \mathrm{dd}(14.1,7.5)$ & 28.0 & 1.94, brd (14.1) \\
\hline & & $1.45, \mathrm{dd}(14.1,7.5)$ & & $1.46, \mathrm{dd}(14.1,7.2)$ \\
\hline 22 & 122.7 & $4.88, \mathrm{t}(7.5)$ & 122.7 & $4.89, \mathrm{t}(7.2)$ \\
\hline 23 & 133.3 & & 133.2 & \\
\hline 24 & 25.7 & $1.55, \mathrm{~s}$ & 25.7 & $1.55, \mathrm{~s}$ \\
\hline 25 & 17.8 & $1.43, \mathrm{~s}$ & 17.8 & $1.43, \mathrm{~s}$ \\
\hline \multirow[t]{2}{*}{26} & 31.5 & $2.74, \mathrm{dd}(14.4,8.5)$ & 31.5 & $2.74, \mathrm{dd}(14.4,8.5)$ \\
\hline & & $2.65, \mathrm{dd}(14.4,8.5)$ & & $2.66, \mathrm{dd}(14.4,8.5)$ \\
\hline 27 & 119.9 & $5.68, \mathrm{t}(8.5)$ & 119.9 & $5.68, \mathrm{t}(7.8)$ \\
\hline 28 & 133.4 & & 133.4 & \\
\hline 29 & 26.1 & $1.66, \mathrm{~s}$ & 26.1 & $1.66, \mathrm{~s}$ \\
\hline 30 & 17.8 & $1.55, \mathrm{~s}$ & 17.8 & $1.55, \mathrm{~s}$ \\
\hline \multicolumn{2}{|c|}{ OH-5 } & $8.41, \mathrm{~s}$ & & $8.39, \mathrm{~s}$ \\
\hline
\end{tabular}

${ }^{a}$ There was an apparent typo in the original assignment (0.73), and the real chemical shift of H12 of SE (2a) should be 0.78). 


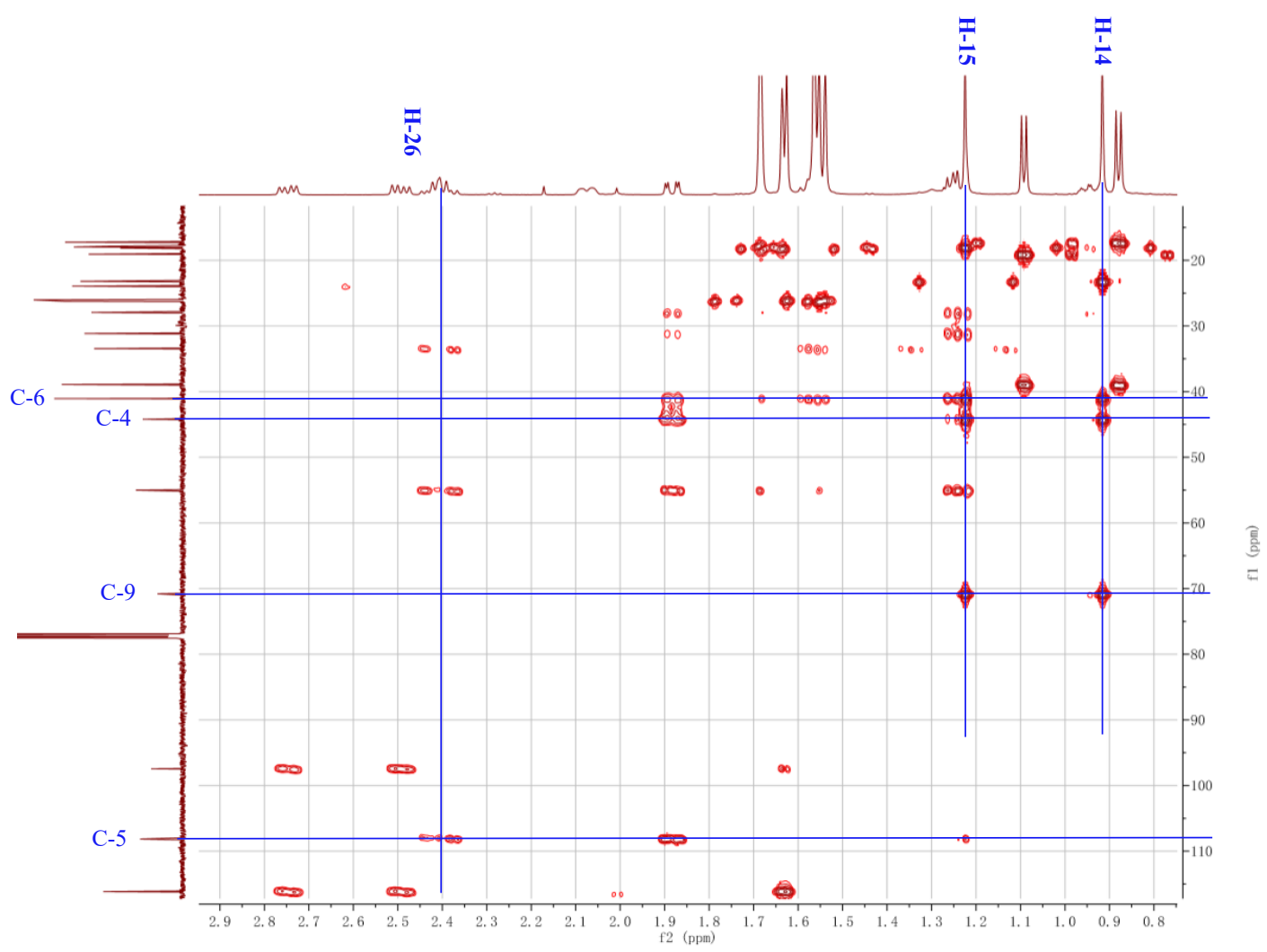

Figure S1. Portion of the HMBC spectrum of SE revealing strong correlations from both $\mathrm{H} 14$ and $\mathrm{H} 15$ to $\mathrm{C} 4, \mathrm{C} 6$, and C9, and from $\mathrm{H} 26$ to $\mathrm{C} 5$. 


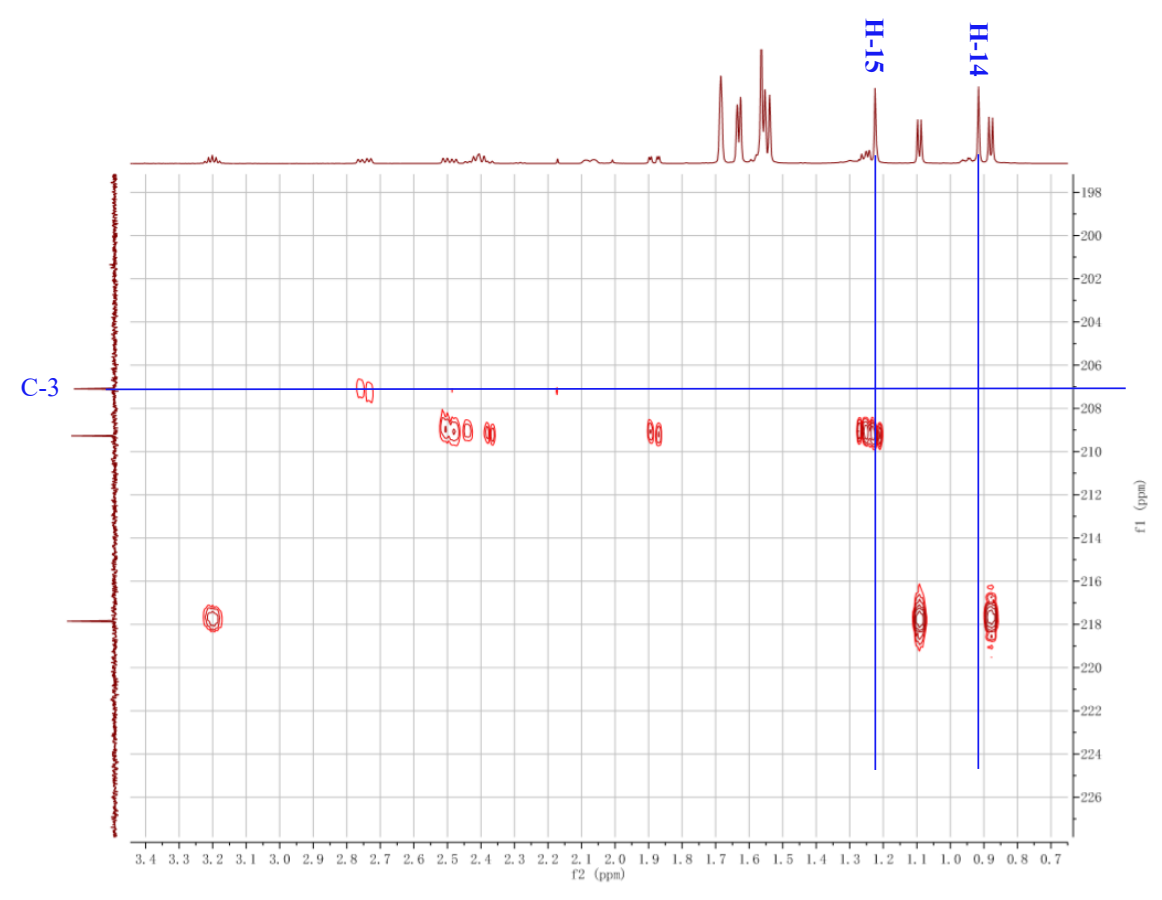

Figure S2. Portion of the HMBC spectrum of SE illustrating the absent correlations of H14-C3 and H15-C3. 

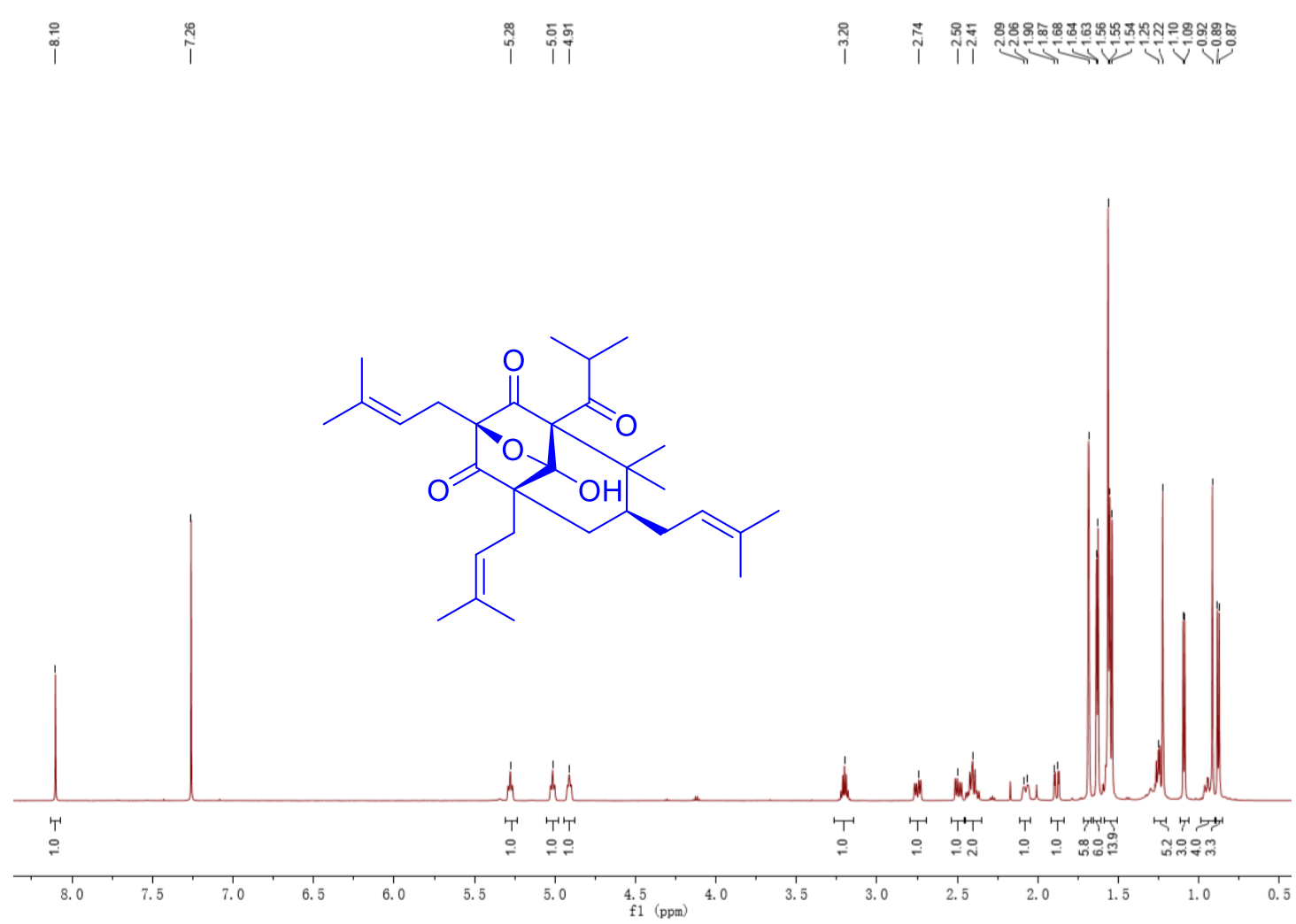

Figure S3. ${ }^{1} \mathrm{H}$ NMR spectrum of $\mathrm{SE}$ in $\mathrm{CDCl}_{3}$.
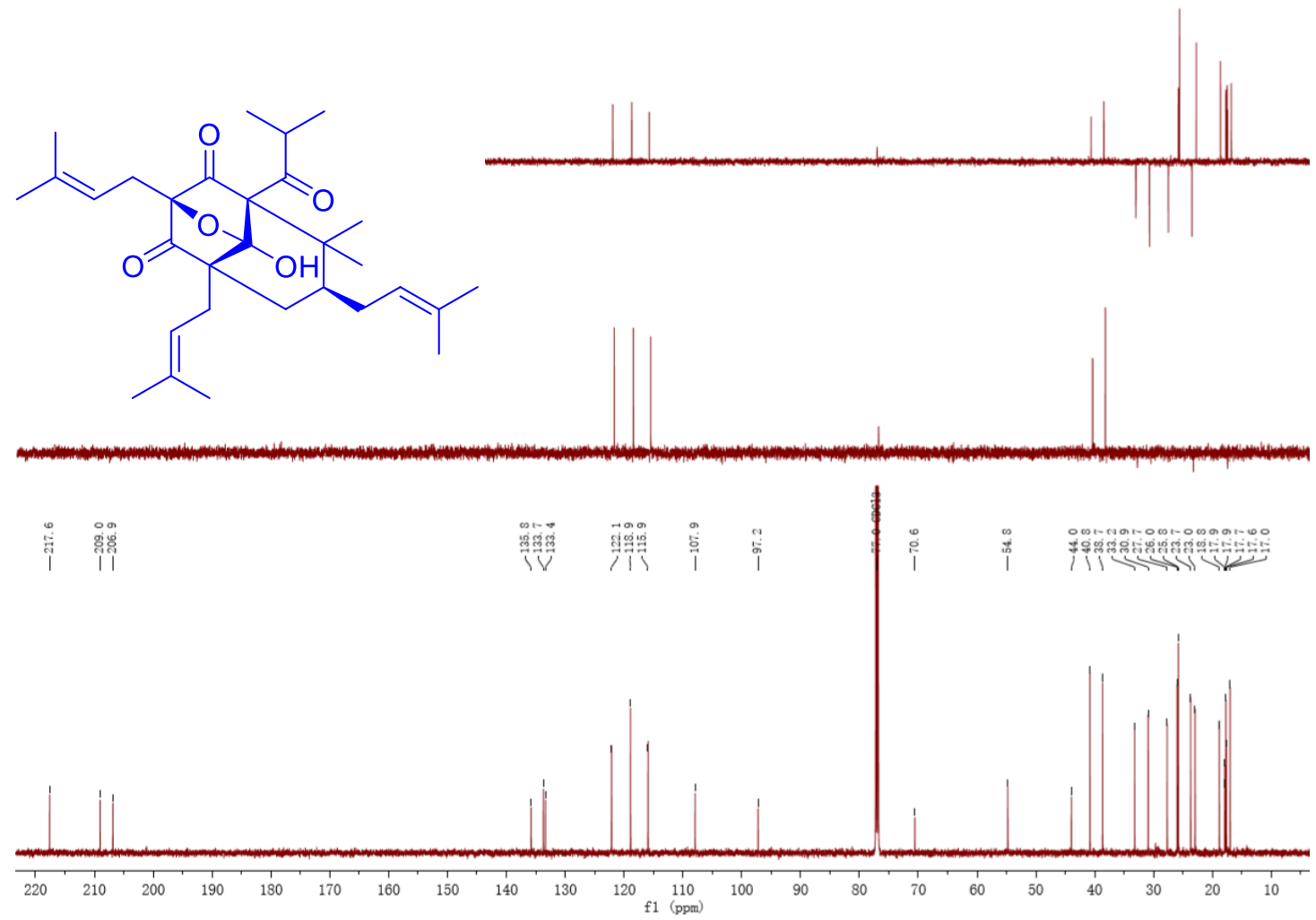

Figure S4. ${ }^{13} \mathrm{C}$ and DEPT spectra of SE in $\mathrm{CDCl}_{3}$. 


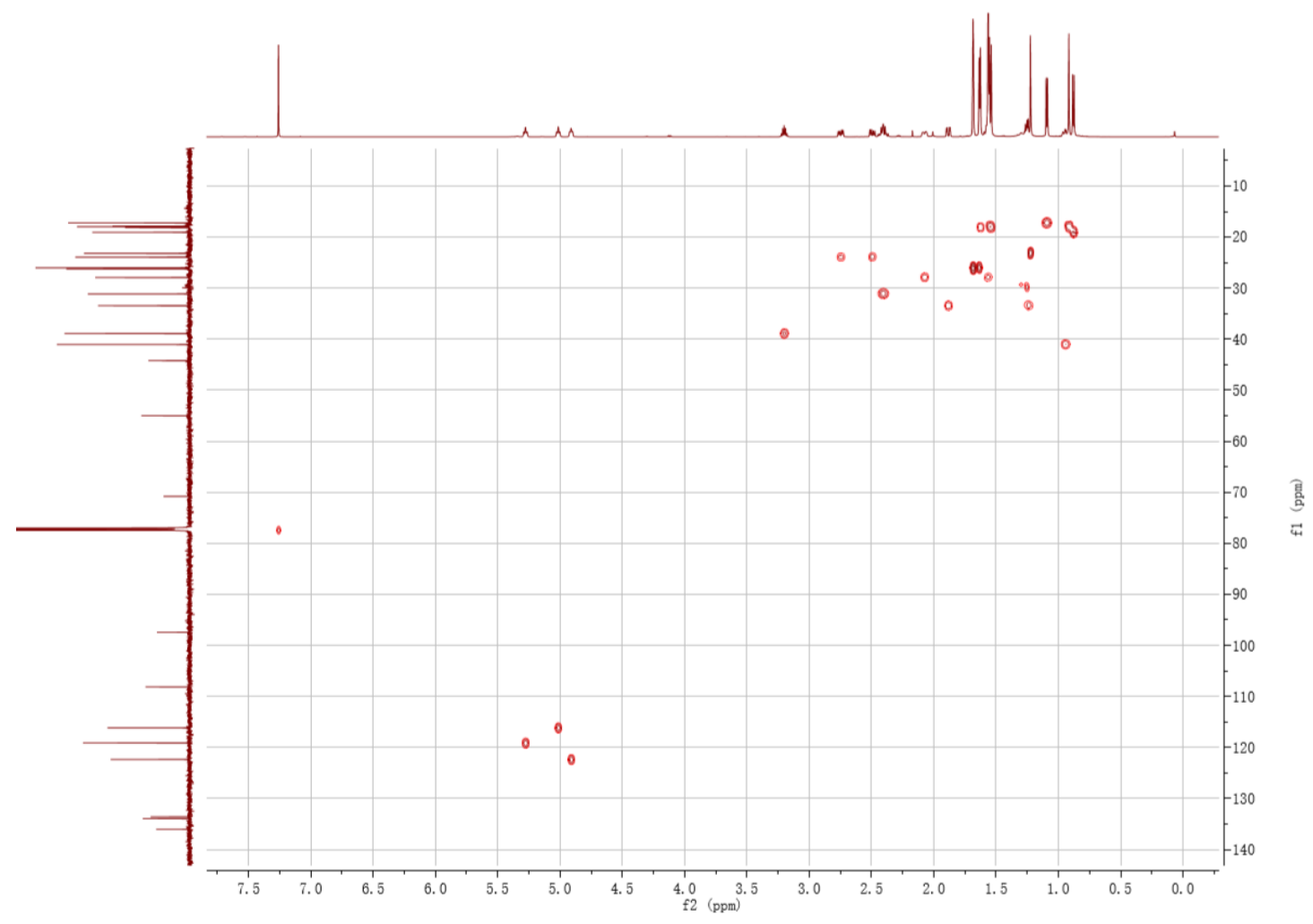

Figure S5. HSQC spectrum of $\mathrm{SE}$ in $\mathrm{CDCl}_{3}$.

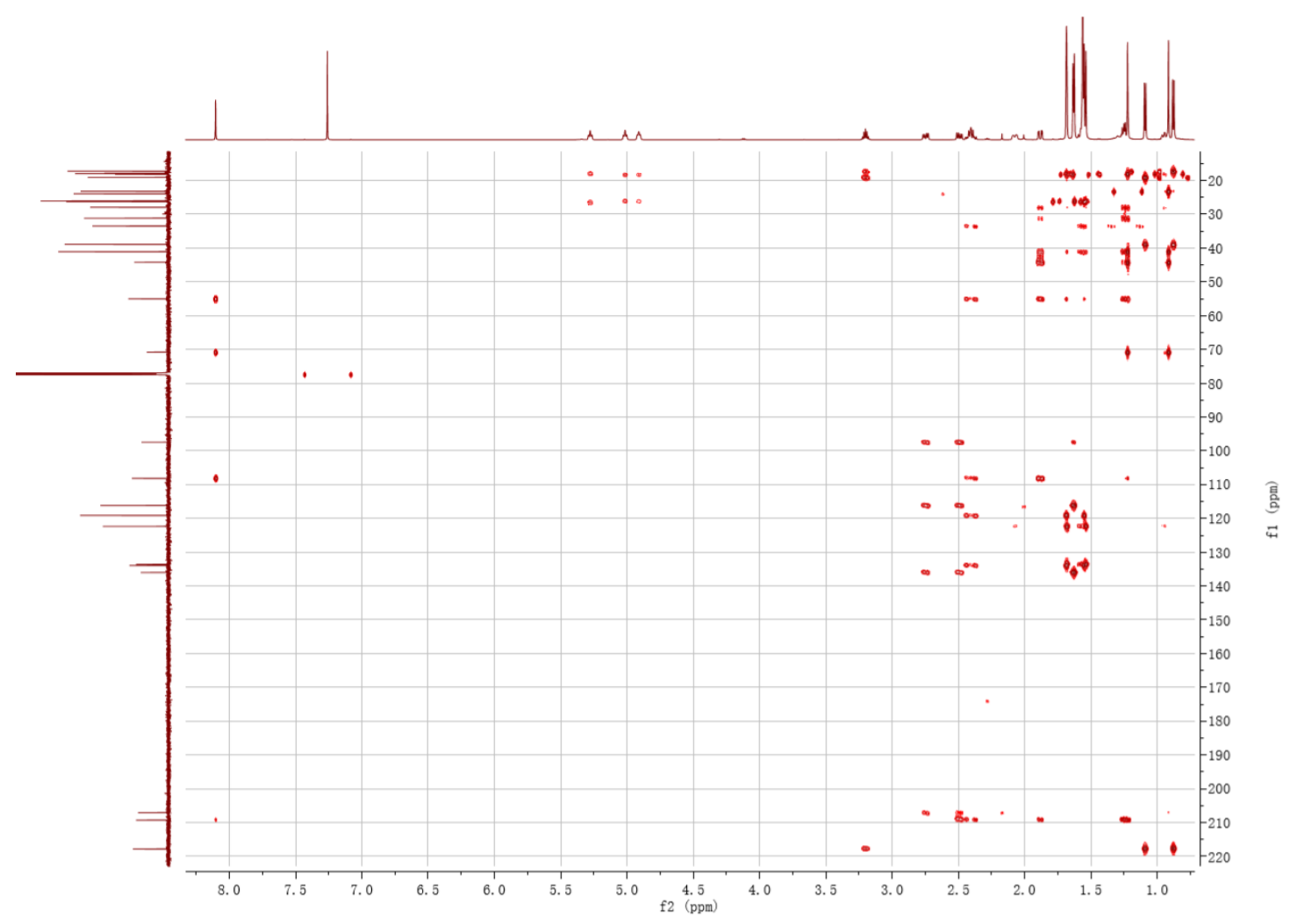

Figure S6. $\mathrm{HMBC}$ spectrum of $\mathrm{SE}$ in $\mathrm{CDCl}_{3}$. 


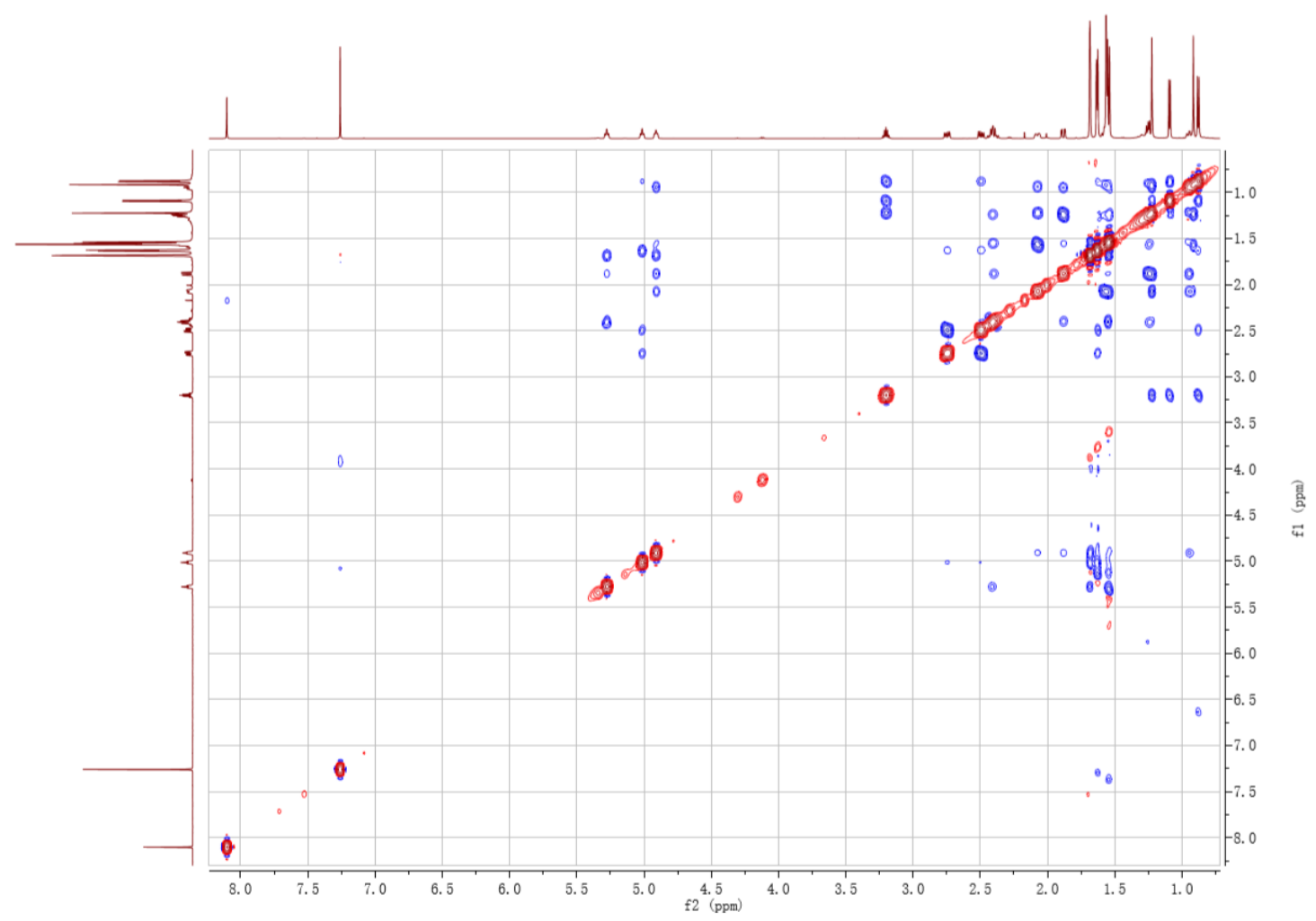

Figure S7. ROESY spectrum of SE in $\mathrm{CDCl}_{3}$.

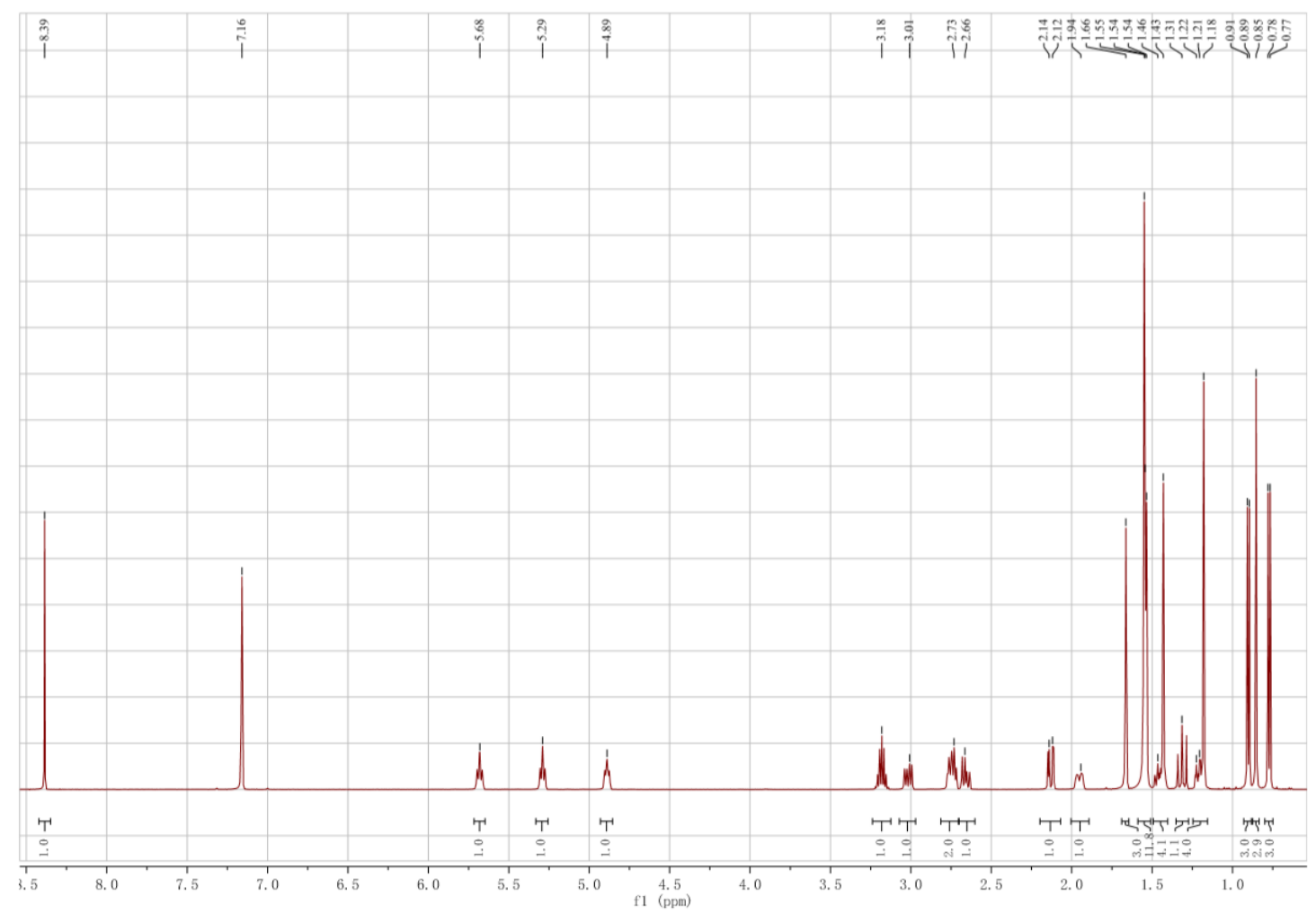

Figure S8. ${ }^{1} \mathrm{H}$ NMR spectrum of $\mathrm{SE}$ in $\mathrm{C}_{6} \mathrm{D}_{6}$. 


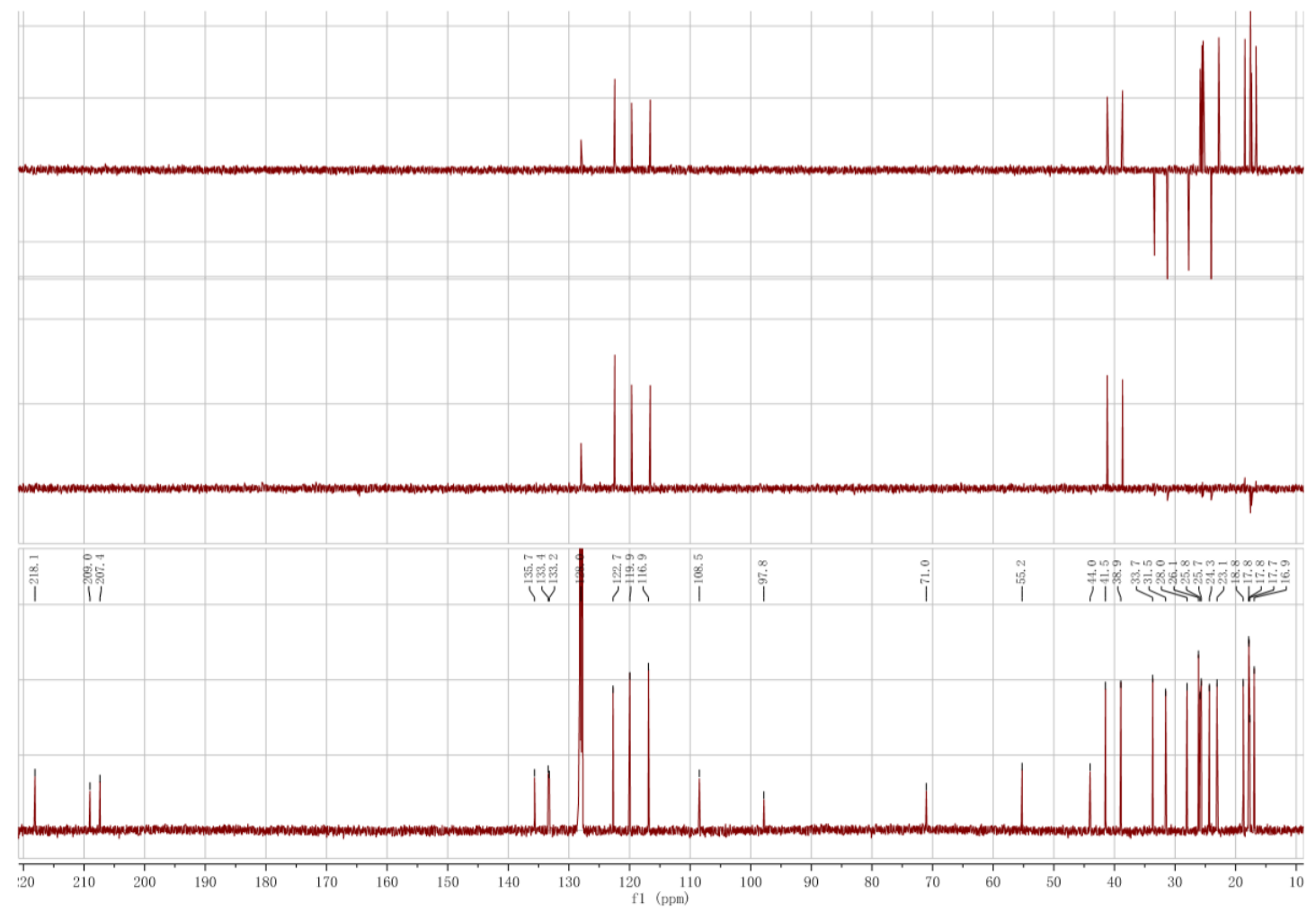

Figure S9. ${ }^{13} \mathrm{C}$ and DEPT spectra of SE in $\mathrm{C}_{6} \mathrm{D}_{6}$.

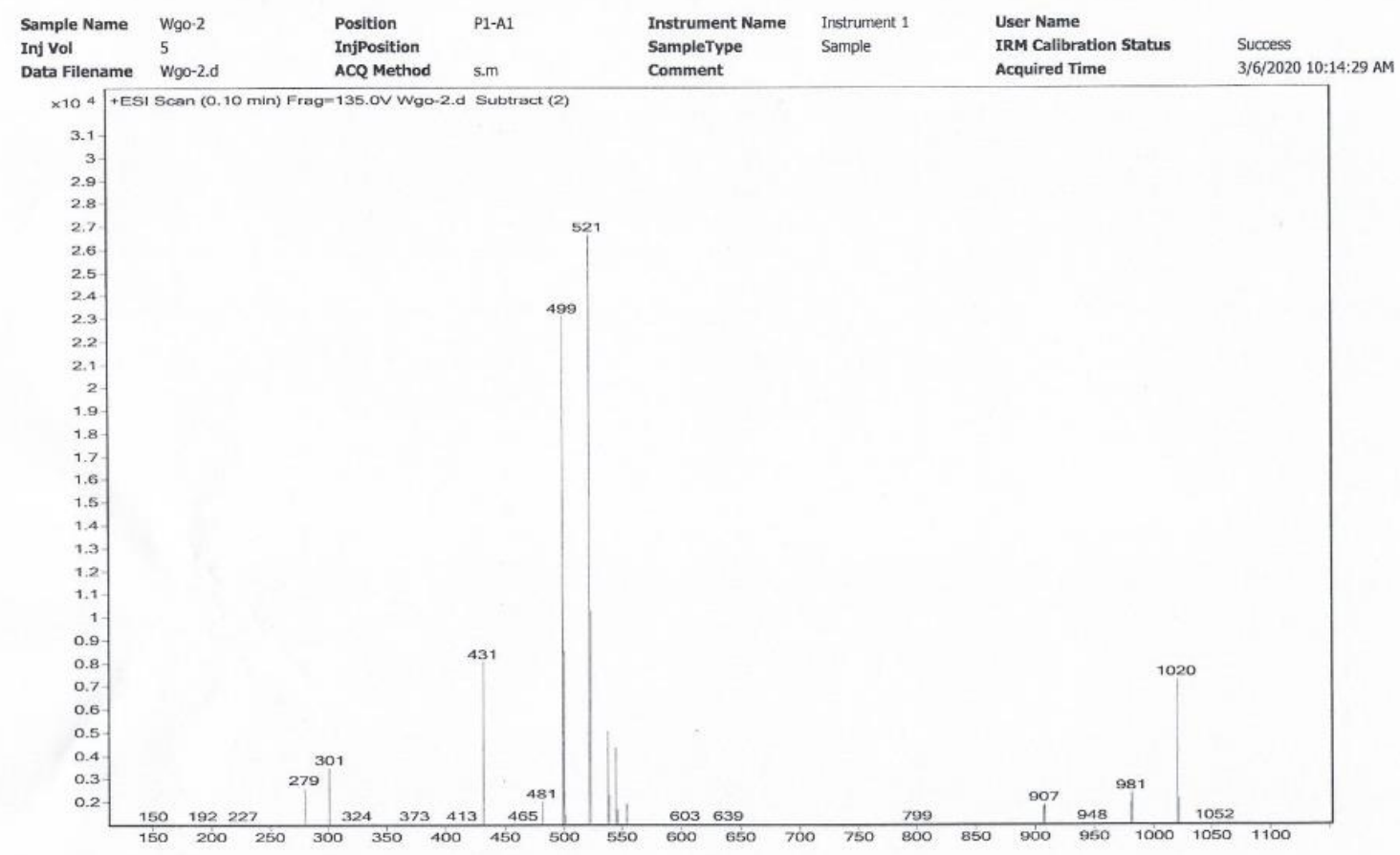

Figure S10. ESIMS spectrum of GT. 
Qualitative Analysis Report

\begin{tabular}{|c|c|c|c|}
\hline Data Filename & Wgo-2.d & Sample Name & Wgo-2 \\
\hline Sample Type & Sample & Position & P1-A1 \\
\hline Instrument Name & Instrument 1 & User Name & \\
\hline Acq Method & s.m & Acquired Time & 3/6/2020 10:14:29 AM \\
\hline IRM Calibration Status & Success & DA Method & Default.m \\
\hline \multicolumn{4}{|l|}{ Comment } \\
\hline Sample Group & & & \\
\hline $\begin{array}{l}\text { Acquisition SW } \\
\text { Version }\end{array}$ & $\begin{array}{l}\text { ies TOF/6500 series } \\
05.01(B 5125.2)\end{array}$ & & \\
\hline
\end{tabular}

User Spectra

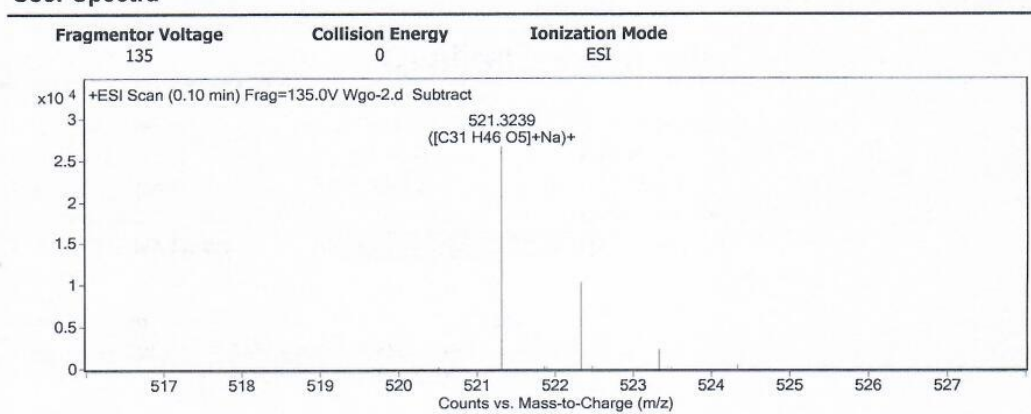

Peak List

\begin{tabular}{|l|l|l|l|l|}
\hline $\boldsymbol{m} / \boldsymbol{z}$ & $\mathbf{z}$ & Abund & Formula & Ion \\
\hline 301.1406 & 1 & 4518.4 & & \\
\hline 431.2793 & 1 & 8021.2 & & \\
\hline 499.3419 & 1 & 23082.95 & & \\
\hline 500.3454 & 1 & 8505.43 & & \\
\hline 521.3239 & 1 & 26624.57 & $\mathrm{C} 31 \mathrm{H} 46$ 05 & $(\mathrm{M}+\mathrm{Na})+$ \\
\hline 522.327 & 1 & 10274.15 & $\mathrm{C} 31 \mathrm{H} 46$ 05 & $(\mathrm{M}+\mathrm{Na})+$ \\
\hline 537.2998 & 1 & 5058.01 & & \\
\hline 544.3993 & 1 & 4317.4 & & \\
\hline 1019.6568 & 1 & 7244.92 & & \\
\hline 1020.6608 & 1 & 5214.15 & & \\
\hline
\end{tabular}

\begin{tabular}{l|c|c|c}
1020.6608 & 1 & 5214.15 & \\
\hline Formula Calculator Element Limits
\end{tabular}

\begin{tabular}{|l|l|l|}
\hline Element & Min & Max \\
\hline
\end{tabular}

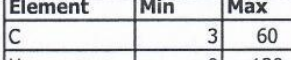

\begin{tabular}{|l|c|c|}
\hline $\mathrm{C}$ & 3 & 60 \\
\hline $\mathrm{H}$ & 0 & 30 \\
\hline
\end{tabular}

Formula Calculator Results

\begin{tabular}{|l|l|l|l|l|l|l|}
\hline Formula & CalculatedMass & CalculatedMz & Mz & Diff. (mDa) & Diff. (ppm) & DBE \\
\hline
\end{tabular}

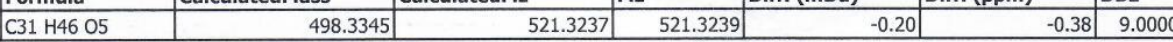

--- End Of Report ---

Figure S11. HRESIMS spectrum of GT. 


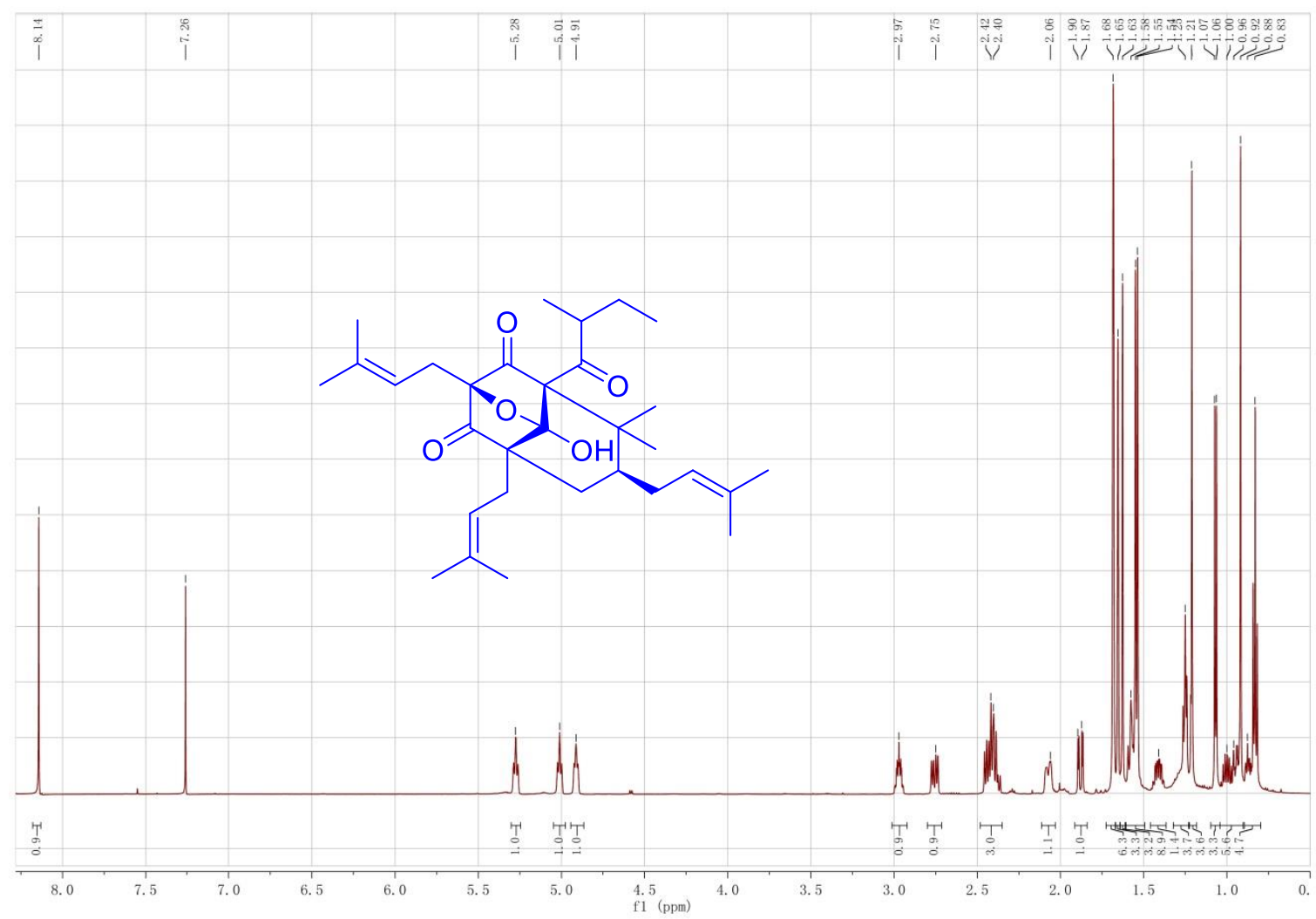

Figure S12. ${ }^{1} \mathrm{H}$ NMR spectrum of GT in $\mathrm{CDCl}_{3}$.
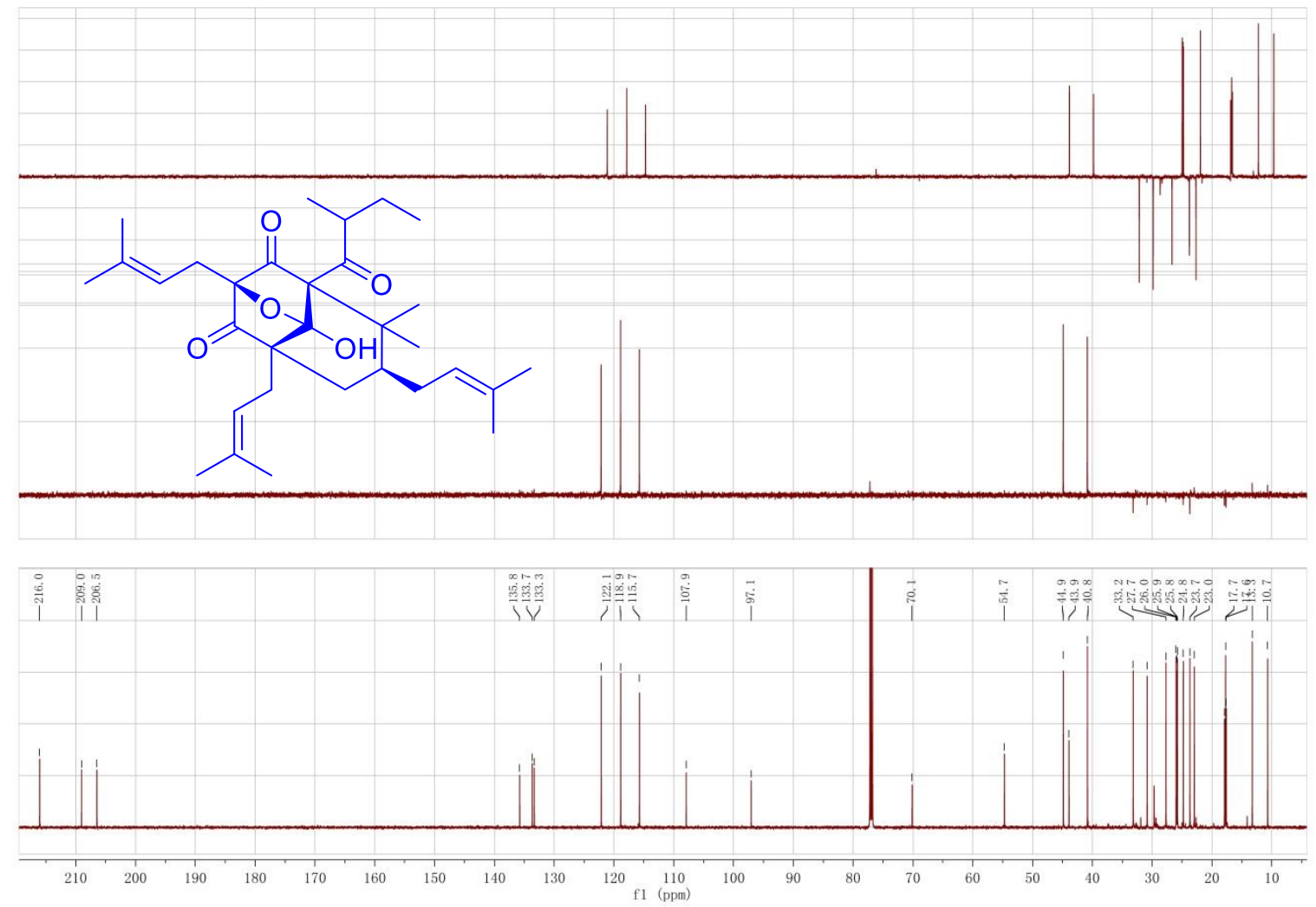

Figure S13. ${ }^{13} \mathrm{C}$ and DEPT spectra of GT in $\mathrm{CDCl}_{3}$. 


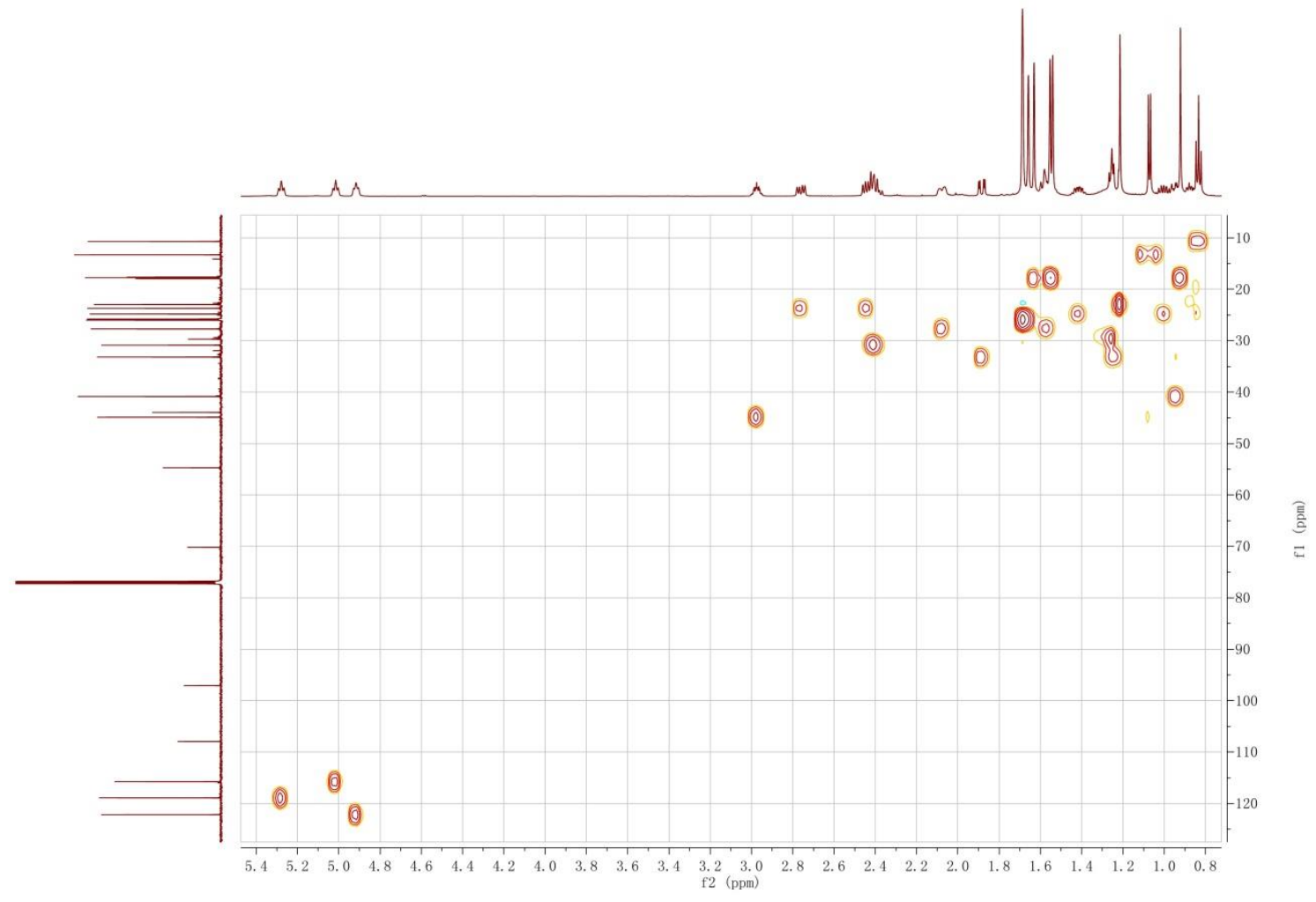

Figure S14. HSQC spectrum of GT.

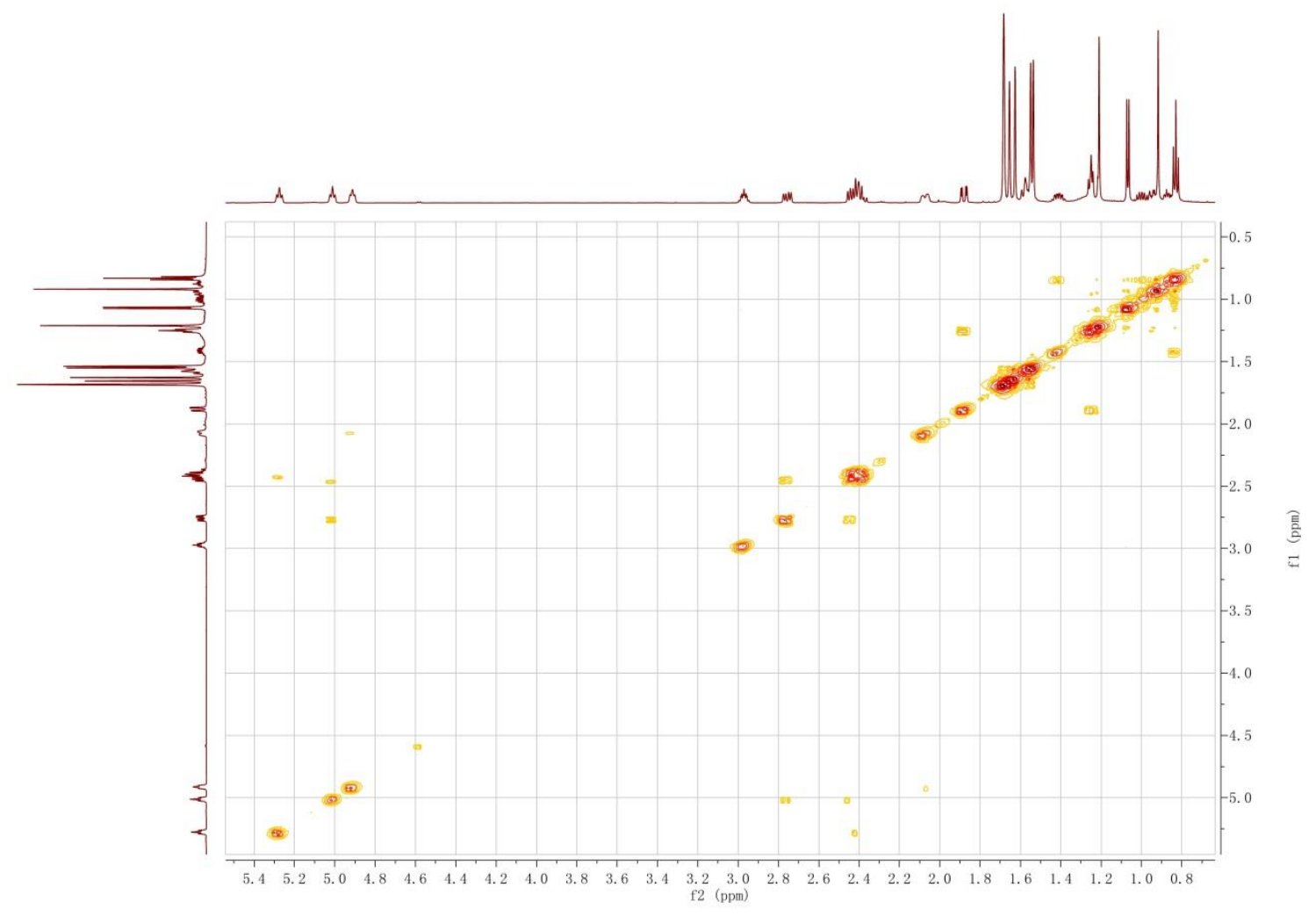

Figure S15. ${ }^{1} \mathrm{H}-{ }^{1} \mathrm{H}$ COSY spectrum of GT. 


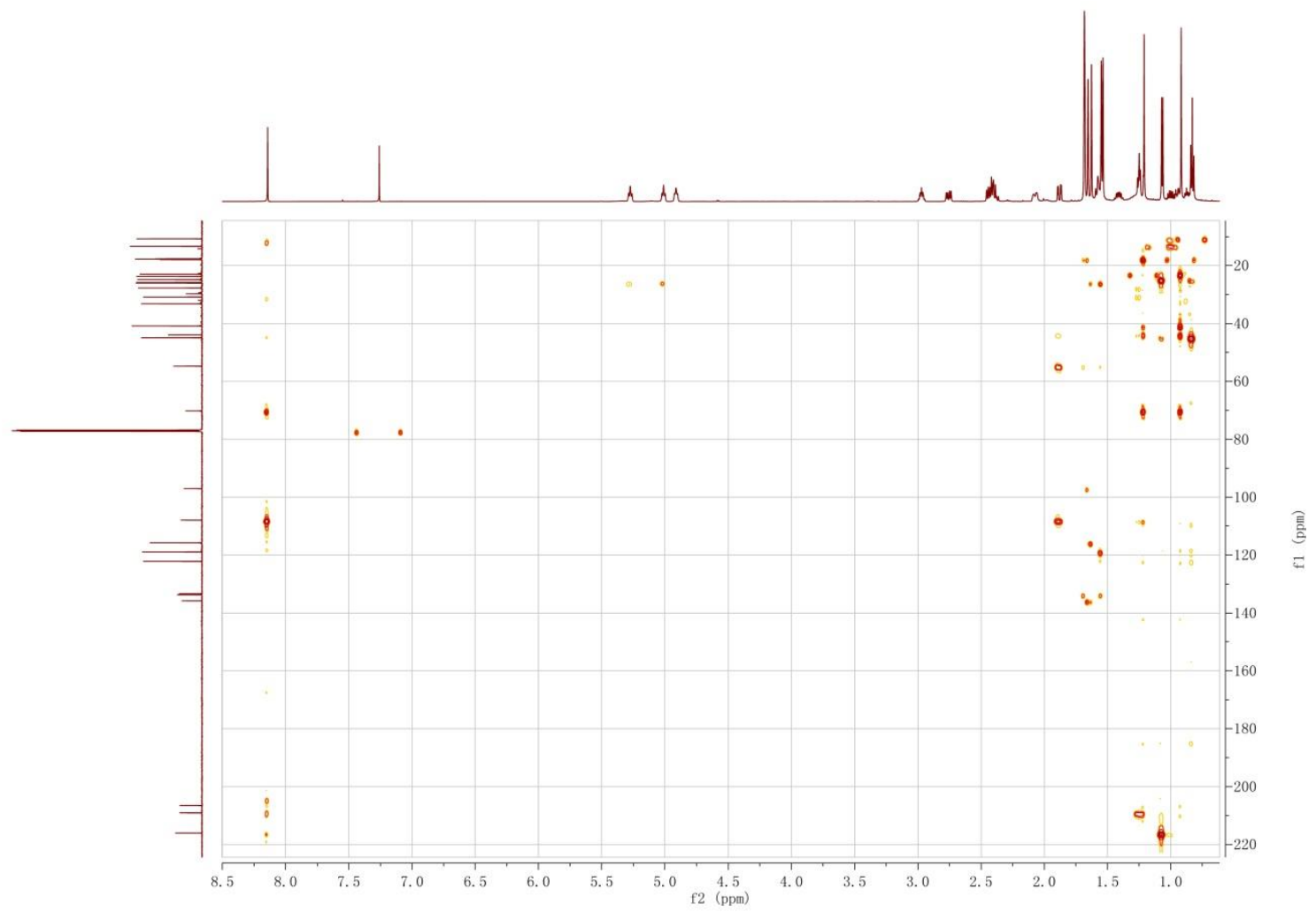

Figure S16. HMBC spectrum of GT.

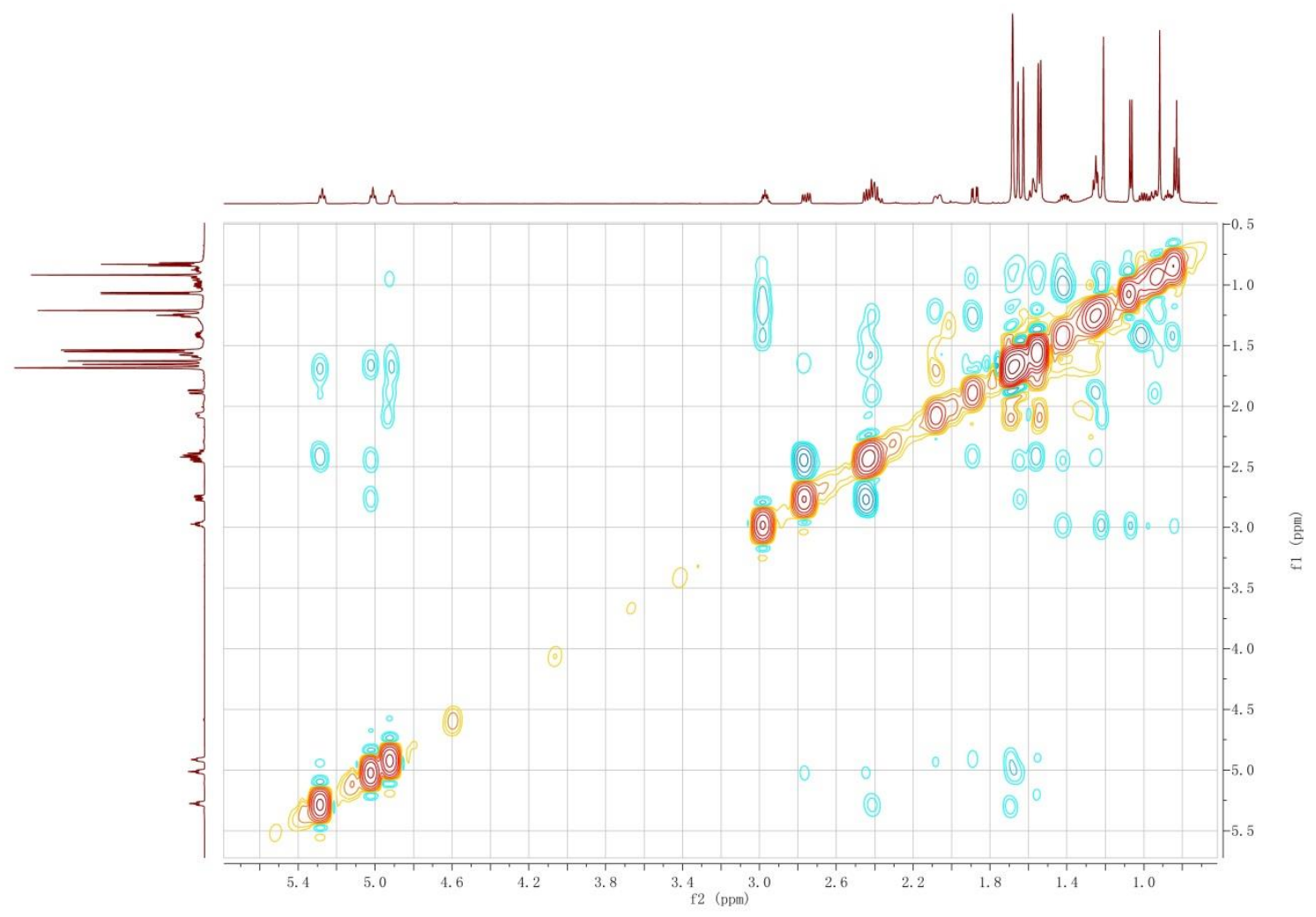

Figure S17. NOESY spectrum of GT. 


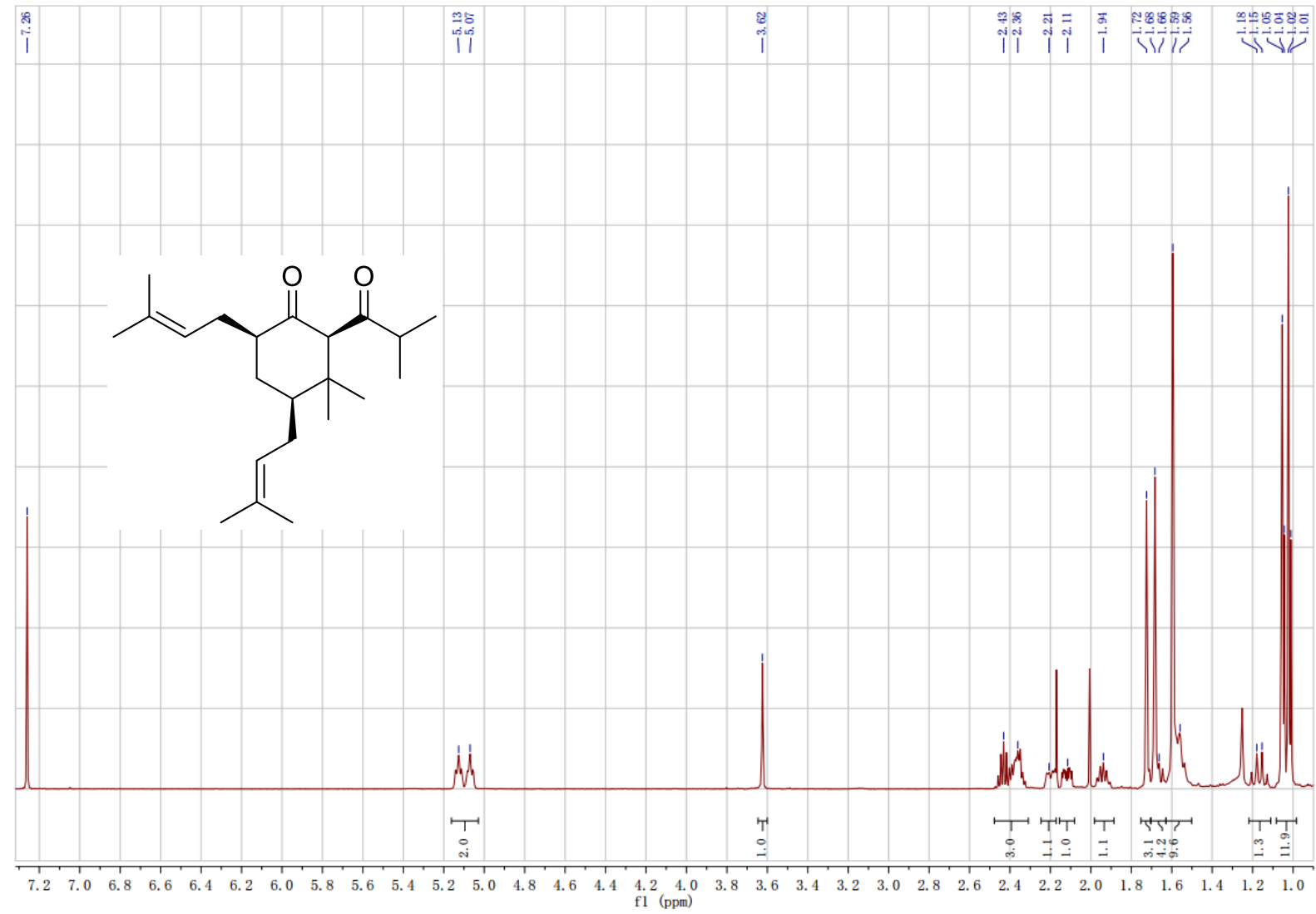

Figure S18. ${ }^{1} \mathrm{H}$ NMR spectrum of GN (4) in $\mathrm{CDCl}_{3}$.
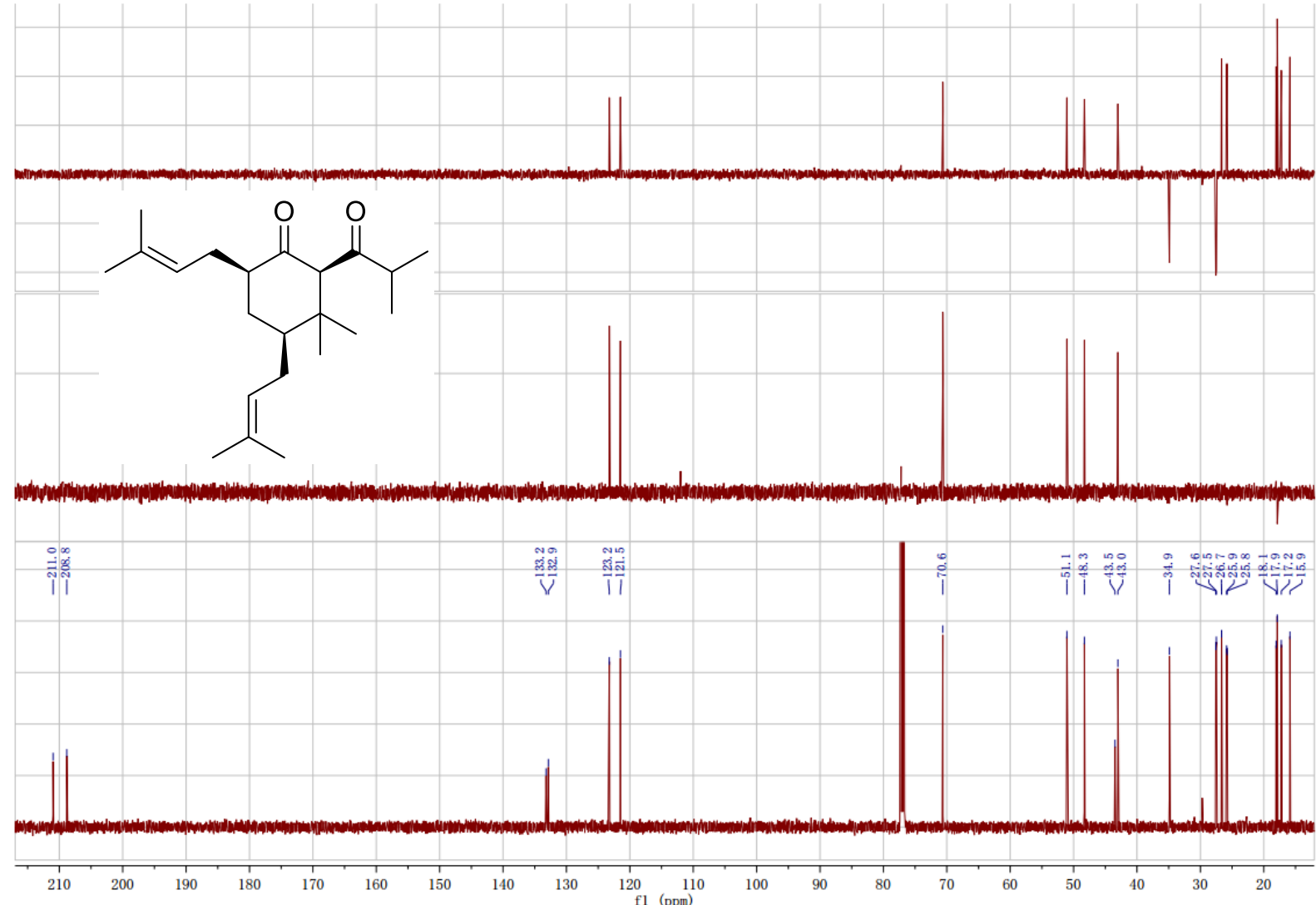

Figure S19. ${ }^{13} \mathrm{C}$ and DEPT spectra of $\mathrm{GN}(4)$ in $\mathrm{CDCl}_{3}$. 